\title{
Effectiveness of multifaceted implementation strategies for the implementation of back and neck pain guidelines in health care: a systematic review
}

Arnela Suman $^{1}$ (D), Marije F. Dikkers ${ }^{2}$, Frederieke G. Schaafsma ${ }^{1,3^{*}}$, Maurits W. van Tulder ${ }^{2}$ and Johannes R. Anema ${ }^{1,3}$

\begin{abstract}
Background: For the optimal use of clinical guidelines in daily practice, mere distribution of guidelines and materials is not enough, and active implementation is needed. This review investigated the effectiveness of multifaceted implementation strategies compared to minimal, single, or no implementation strategy for the implementation of non-specific low back and/or neck pain guidelines in health care.

Methods: The following electronic databases were searched from inception to June 1, 2015: MEDLINE, Embase, Psyclnfo, the Cochrane Library, and CINAHL. The search strategy was restricted to low back pain, neck pain, and implementation research. Studies were included if their design was a randomized controlled trial, reporting on patients (age $\geq 18$ years) with non-specific low back pain or neck pain (with or without radiating pain). Trials were eligible if they reported patient outcomes, measures of healthcare professional behaviour, and/or outcomes on healthcare level. The primary outcome was professional behaviour. Guidelines that were evaluated in the studies had to be implemented in a healthcare setting. No language restrictions were applied, and studies had to be published full-text in peer-reviewed journals, thus excluding abstract only publications, conference abstracts, and dissertation articles. Two researchers independently screened titles and abstract, extracted data from included studies, and performed risk of bias assessments.
\end{abstract}

Results: After removal of duplicates, the search resulted in 4750 abstracts to be screened. Of 43 full-text articles assessed for eligibility, 12 were included in this review, reporting on 9 individual studies, and separate cost-effectiveness analyses of 3 included studies. Implementation strategies varied between studies. Meta-analyses did not reveal any differences in effect between multifaceted strategies and controls.

(Continued on next page)

\footnotetext{
* Correspondence: f.schaafsma@vumc.nl

${ }^{1}$ Department of Public and Occupational Health, VU University medical

centre and the EMGO+ Institute for Health and Care Research, PO Box 7057,

1007, MB, Amsterdam, The Netherlands

${ }^{3}$ Research Centre for Insurance Medicine, Collaboration between

AMC-UMCG-UWV-VUmc, Amsterdam, The Netherlands

Full list of author information is available at the end of the article
}

(c) 2016 The Author(s). Open Access This article is distributed under the terms of the Creative Commons Attribution 4.0 International License (http://creativecommons.org/licenses/by/4.0/), which permits unrestricted use, distribution, and reproduction in any medium, provided you give appropriate credit to the original author(s) and the source, provide a link to the Creative Commons license, and indicate if changes were made. The Creative Commons Public Domain Dedication waiver (http://creativecommons.org/publicdomain/zero/1.0/) applies to the data made available in this article, unless otherwise stated. 
(Continued from previous page)

Conclusion: This review showed that multifaceted strategies for the implementation of neck and/or back pain guidelines in health care do not significantly improve professional behaviour outcomes. No effects on patient outcomes or cost of care could be found. More research is necessary to determine whether multifaceted implementation strategies are conducted as planned and whether these strategies are effective in changing professional behaviour and thereby clinical practice.

Keywords: Systematic review, Multifaceted implementation strategies, Guideline implementation, Non-specific low back pain

\section{Introduction}

The recent Global Burden of Disease Study showed that low back pain (LBP), with 83 million years lived with disability, is the leading cause of disability worldwide, while neck pain (NP) is ranked 4th with 33.6 million years lived with disability [1-3]. To assist healthcare professionals in providing best-evidence care for LBP and NP, many guidelines for these health problems have been developed $[4,5]$. Clinical practice guidelines are defined by the Institute of Medicine as 'statements that include recommendations intended to optimize patient care that are informed by a systematic review of evidence and an assessment of the benefits and harms of alternative care options, and are aimed at improving healthcare quality and outcomes' [6]. Most of the guidelines for LBP/NP are developed for multidisciplinary use in primary care and are mainly national professional guidelines. The contents of these guidelines are similar. For example, the guidelines encourage similar diagnostic triages and discourage the use of diagnostic imaging, bed rest, and referrals to specialist care unless neurological or pathological causes are suspected [4]. The use of these guidelines might improve the quality of care for patients with LBP/NP and reduce the financial and societal burden of these disorders.

For the optimal use of guidelines in clinical practice, mere distribution of the guideline and information materials among healthcare professionals is not enough and active implementation is a necessity [7]. Many studies have been conducted to investigate the effectiveness of implementation strategies. For example, the Effective Practice and Organisation of Care (EPOC) Group of the Cochrane Collaboration has published several systematic reviews on this topic. The results of various implementation strategies, such as the use of educational meetings and workshops, educational outreach, and audit and feedback have shown small effects on improvement of professional practice $(6 \%$ improvement for educational meetings and outreach and $5 \%$ for audit and feedback) [8-10]. In line with these findings, Grol and Wensing argued that the simultaneous use of several implementation strategies, i.e. a multifaceted or multicomponent approach to implementation is most effective in successfully implementing guidelines and thus changing practice [7]. However, a recent overview of systematic reviews of multifaceted implementation strategies by Squires et al. suggested that these strategies may not be more effective than single-component interventions [11].

As no studies up to now specifically reviewed the effect of multifaceted implementation strategies for the implementation of non-specific LBP/NP guidelines, the current systematic review will address the following research question: 'What is the effectiveness of multifaceted implementation strategies compared to minimal, single or no implementation strategy for the implementation of non-specific low back and/or neck pain guidelines in health care?' Outcomes on healthcare professional behaviour (e.g. referral for diagnostic imaging), and patient health (e.g. quality of life) will be assessed to measure adherence to the guidelines and thereby the success of the implementation process.

\section{Material and methods Eligibility criteria}

For the purpose of this review, multifaceted strategies were defined as interventions that consist of a combination of two or more elements from the implementation strategy taxonomy of the EPOC classification system [12]. As this review aimed to assess the effectiveness of implementation strategies, studies were considered for this review if their design was a randomized controlled trial (RCT), involving either individual or cluster randomization and including a control group that received a minimal, single, or no implementation strategy. Studies were eligible if reporting on patients of either gender (age $\geq 18$ years) with non-specific LBP or NP (with or without radiating pain) of any duration. Studies of LBP or NP caused by infection, cauda equina syndrome, bone rarefaction, compression fracture of a vertebral body, tumour, or fibromyalgia were excluded. Cost-effectiveness analyses of included trials were also included. Trials were eligible if they reported measures of healthcare professional behaviour (the primary outcome for this review, patient outcomes, and/or outcomes on healthcare level). Guidelines that were evaluated in the studies had to be implemented in a healthcare setting (i.e. a setting where individual health 
care is provided to a patient), for example, primary care (general practitioner (GP) or physiotherapist (PT)), occupational health care, or secondary (hospital) care. Guidelines for healthcare insurance were therefore excluded. No language restrictions were applied, and studies had to be published full-text in peer-reviewed journals, thus excluding abstract only publications, conference abstracts, and dissertation articles.

\section{Information sources}

The following electronic databases were searched until June 1, 2015: MEDLINE (PubMed), Embase, PsycInfo, the Cochrane Library, and CINAHL (Ebsco). In close collaboration with a medical information specialist, a broad search was performed with only two restrictions: LBP and/or NP and implementation. Full electronic search strategies for all five databases are presented in Additional file 1: Appendix A. In cases of ambiguity, or where full-text publications of selected abstracts could not be found, authors of the respective studies were contacted. The reference lists of all included studies were screened to identify additional studies.

\section{Study selection and data extraction}

After removal of duplicate results, two reviewers (AS and MD) independently reviewed all titles and abstracts identified by the electronic search. Subsequently, the reviewers engaged in a consensus method to eliminate discrepancies in the selection process. In cases where the reviewers could not come to consensus regarding study eligibility, a third reviewer (FGS or MWvT) was consulted. Full-text articles of studies were obtained when the study was deemed to meet the inclusion criteria or in cases where perusal of title and abstract did not provide sufficient information to assess eligibility of the study. Both reviewers independently screened all selected full-text articles for definitive eligibility, and the same consensus protocol was followed as for the screening of titles and abstracts. Using an adapted form of the 'Good practice data extraction form' of the EPOC group, study characteristics and relevant data of all included studies were independently extracted by the two reviewers (AS and MD). Results were discussed in order to reach consensus and assure correct interpretation of the studies. In cases where consensus could not be reached, a third reviewer (FGS) was consulted.

\section{Assessment of risk of bias of studies}

The risk of bias of the included randomized trials was evaluated by two review authors independently (AS and $\mathrm{MD})$, using the Cochrane Collaboration's tool for assessing risk of bias and the suggested risk of bias criteria for EPOC reviews [13, 14]. Disagreements were resolved by consensus. The quality of the economic evaluations was not assessed, because this was outside the scope of the current review. The following criteria were assessed for high, unclear or low risk of bias for every study: random sequence generation (selection bias); allocation concealment (selection bias); similarity of baseline characteristics and outcome measurements; follow-up; blinding of participants and personnel (performance bias); blinding of outcome assessment (detection bias); protection against contamination; incomplete outcome data (attrition bias); selective outcome reporting (reporting bias); and other bias.

Studies that had a low risk of bias score on at least six criteria were judged to be low risk of bias studies [15]. Studies that had five or less low risk of bias scores were judged to be high risk of bias studies.

Two review authors (AS and MD) independently assessed the overall quality of the evidence for all pooled outcomes using the Grading of Recommendations Assessment, Development and Evaluation (GRADE) approach [16-19]. The GRADE approach specifies four levels of quality. High-quality rating is for randomized controlled evidence, and the quality rating can be downgraded if limitations in one or more of the following domains are encountered: Study limitations encountered in 'risk of bias' assessment of study; consistency of study (i.e. the similarity of estimates of treatment effects for the outcome across studies); directness of the study (i.e. the extent to which the participants, interventions, and outcomes in the studies were comparable to those defined in the inclusion criteria of this review); precision of the study (i.e. the degree of certainty surrounding an effect estimate); and publication bias (i.e. the probability of selective publication of studies and outcomes).

The overall quality of the evidence for each pooled outcome was the result of the combination of all domains and leads to four levels of evidence [18]:

- High-quality evidence: Further research is very unlikely to change the confidence in the estimate of effect.

- Moderate-quality evidence: Further research is likely to have an impact on the confidence in the estimate of effect and may change the estimate.

- Low-quality evidence: Further research is very likely to have an important impact on the confidence in the estimate of effect and is likely to change the estimate.

- Very low-quality evidence: Any estimate of effect is very uncertain.

\section{Data extraction}

Two independent reviewers (AS and MD) extracted data from the included studies using the EPOC data collection checklist and data extraction template [12]. The data 
extraction form was the first pilot tested using one of the included studies. Disagreements in data extraction were resolved by consensus. The following data were extracted:

- Bibliographic data (authors, title study, journal, and date of publication)

- Study characteristics (study type and design, unit of allocation, duration of follow-up)

- Participant characteristics (population description (e.g. (neck or back pain) patients or (type of) professionals), total number of participants randomized, mean age, gender, severity of illness, co-morbidities)

- Setting characteristics (location, social context, clusters, withdrawals, and exclusions)

- Description of intervention and control groups (content, dose, components, duration, timing, delivery, providers, number randomized to group, theory base)

- Outcomes assessed (outcome definitions, time points measured and reported, unit of measurement, outcome tool, scales, missing data)

- Study results (baseline data, comparison, outcome, subgroup, time points, results intervention and comparison)

\section{Synthesis of results}

The included studies first were categorized into types of interventions (according to the EPOC taxonomy) and types of outcome measures. Meta-analyses were separately planned and conducted for the comparison of multifaceted implementation strategies vs. controls (i.e. usual care or minimal implementation) for various outcomes. Outcomes on healthcare professional behaviour were considered indicators for guideline adherence and thus the primary outcome for success of guideline implementation. The effects on professional behaviour were categorized into 'treatment' and 'referral' behaviour outcome groups. In the treatment group, outcomes on treatment behaviour were classified into adequate patient information, advising active treatment, and prescribing medication. Outcomes on referral behaviour were separately analysed for referrals for X-ray, computed tomography $(\mathrm{CT})$, or magnetic resonance imaging (MRI) scans, physical therapy, and speciality/secondary care referrals. Additional file 2: Appendix $\mathrm{C}$ shows the data sources and calculations used for the meta-analyses.

To calculate effects, the data for the pooled outcomes of each study were entered into Review Manager (RevMan) 5.3 software. All pooled outcome data were dichotomous or dichotomized, and for all outcomes, odds

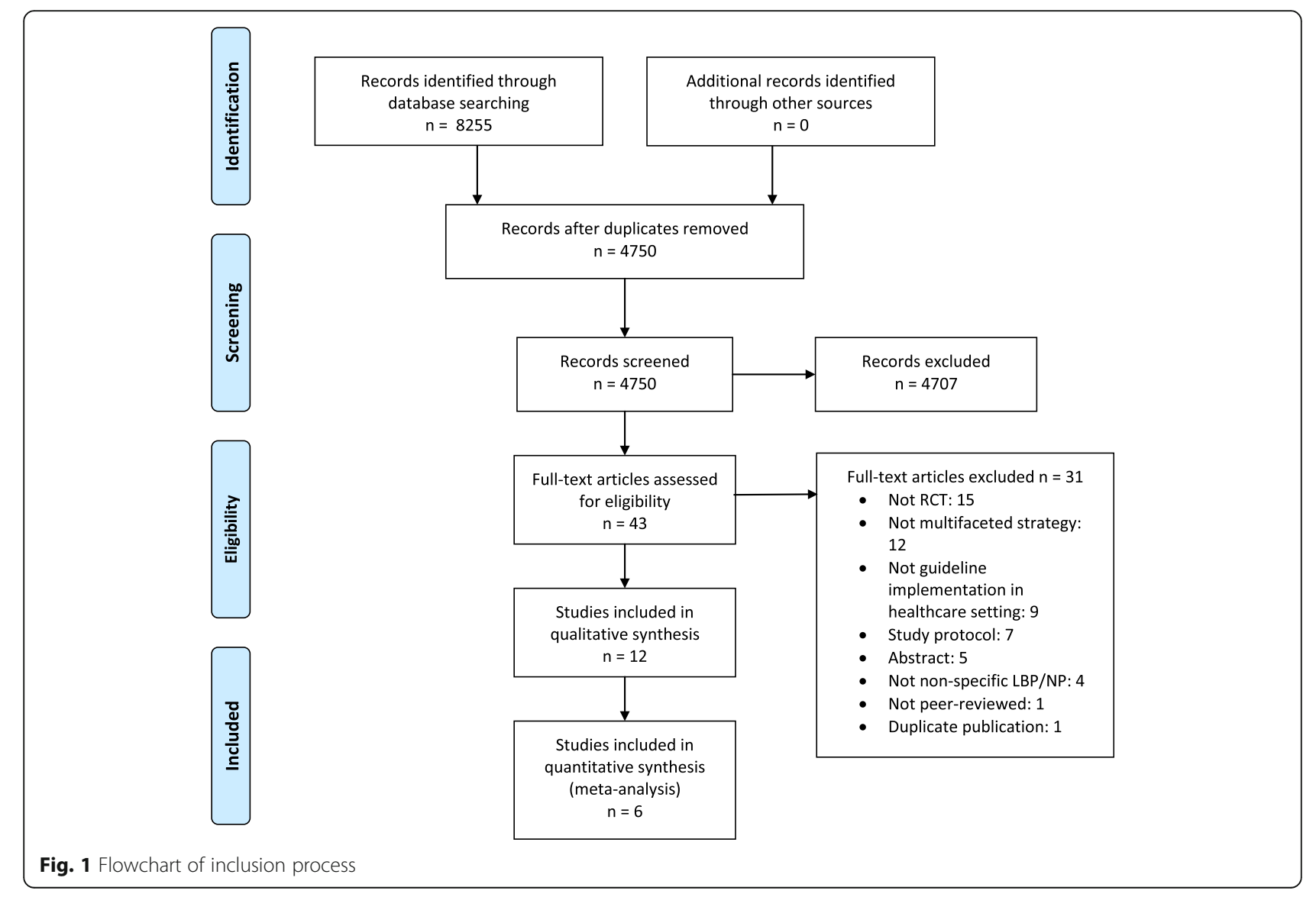


ratios (using random effects models) and $95 \%$ CIs were calculated in RevMan to estimate the implementation effects. To determine the presence of heterogeneity, $I^{2}$ was analysed in RevMan. When $I^{2}$ was more than $50 \%$, the studies were judged to be heterogeneous.

\section{Results}

\section{Identification and selection of studies}

The electronic search resulted in 8255 references, of which 2476 were retrieved from MEDLINE, 4181 from Embase, 876 from CINAHL, 293 from Cochrane, and 429 from PsycInfo. After removing duplicates, titles and abstracts of 4750 records were screened. Of 43 records, full-text articles were screened for eligibility. Twelve articles were included in the current review (see Additional file 3: Table S1) [20-31], and 31 articles were excluded (see Additional file 4: Table S2) [32-62]. Figure 1 shows a flow chart of the inclusion process, including reasons for exclusion (several exclusion reasons per article possible) of records. Screening of reference lists of the included articles did not result in any additional inclusions.

The 12 included articles were based on 8 individual studies, of which 4 based their implementation strategy on theory and described the development of their strategy. Three studies separately reported cost-effectiveness analyses, and 1 study described outcomes on patient and professional levels in 2 separate articles. One study was targeted at patients with a whiplash, while all other studies were on non-specific LBP. Additional file 5: Table S3 provides a summary of the included studies and their characteristics. A great variety of intervention elements was applied across the studies, and several outcomes were measured. The guidelines implemented in the included studies had similar objectives such as encouraging activation, restoration of normal functioning, and exercise, while discouraging referrals for secondary care and diagnostic imaging. Additional file 6: Appendix B lists the excluded full-text articles with reasons for their exclusion.

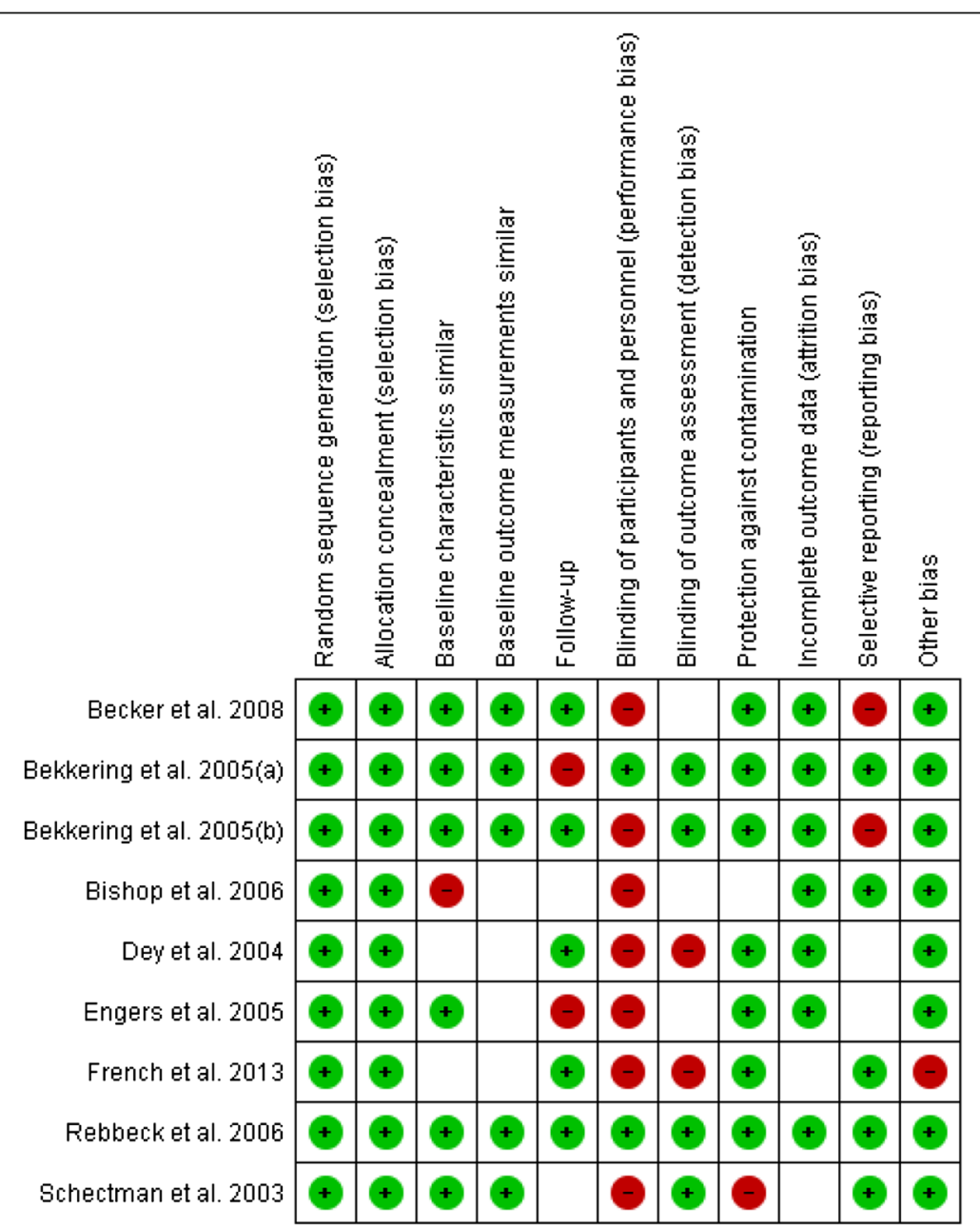

Fig. 2 Risk of bias for included studies 


\begin{tabular}{|c|c|c|c|c|c|c|c|c|c|}
\hline Study or Subgroup & \multicolumn{2}{|c|}{ Intervention } & \multicolumn{2}{|c|}{ Control } & Weight & $\begin{array}{c}\text { Odds Ratio } \\
\text { M-H, Random, } 95 \% \mathrm{Cl}\end{array}$ & \multicolumn{3}{|c|}{$\begin{array}{c}\text { Odds Ratio } \\
\text { M-H, Random, } 95 \% \mathrm{Cl}\end{array}$} \\
\hline Dey et al. 2004 & 158 & 1049 & 156 & 1138 & $31.1 \%$ & $1.12[0.88,1.42]$ & - & 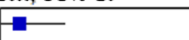 & \\
\hline French et al. 2013 & 643 & 77716 & 768 & 75226 & $45.0 \%$ & $0.81[0.73,0.90]$ & - & & \\
\hline Schectman et al. 2003 & 67 & 828 & 105 & 1218 & $23.9 \%$ & $0.93[0.68,1.29]$ & & & \\
\hline Total $(95 \% \mathrm{Cl})$ & & 79593 & & 77582 & $100.0 \%$ & $0.93[0.75,1.15]$ & & & \\
\hline Total events & 868 & & 1029 & & & & & & \\
\hline $\begin{array}{l}\text { Heterogeneity: } \operatorname{Tau}^{2}=0 \\
\text { Test for overall effect: } Z\end{array}$ & $\begin{array}{l}2 ; \mathrm{Chi}^{2}= \\
0.71(\mathrm{P}=\end{array}$ & $\begin{array}{l}6.13, \mathrm{df}= \\
0.48)\end{array}$ & $=2(\mathrm{P}=0$ & $0.05) ; 1^{2}=$ & $=67 \%$ & & $\begin{array}{cc}0.2 & 0.5 \\
\text { Favours Implementation }\end{array}$ & $\begin{array}{c}2 \\
\text { Favours Control }\end{array}$ & 5 \\
\hline
\end{tabular}

In Additional file 7: Table S4, intervention elements according to the EPOC taxonomy are shown for the included studies. Nine types of elements could be identified. Next to the obvious dissemination of clinical practice guidelines, educational material and educational meetings were the most commonly applied elements. Local opinion leaders, audit and feedback, reminders, and organizational interventions were not used as often, and only three studies applied a patient-mediated intervention element. The implementation strategies of most studies consisted of four to five intervention elements. Additional file 8: Table S5 shows the types of outcomes that were measured in the included studies. Most studies measured physician treatment adherence to guideline recommendations and the number of referrals to secondary care, medical diagnostics, and/or physical therapy. Only three studies reported outcomes on patient level.

\section{Quality of included studies}

Figure 2 shows the risk of bias judgement of the included randomized trials. With only two studies $[25,28]$ judged to have a high risk of bias according to the predefined cut-off point, overall quality of the included studies was good. Blinding of participants and personnel (performance bias) was judged to be a source of high risk of bias in all but two studies [22, 30]. Other sources of bias like a follow-up of at least $80 \%$, blinding of outcome assessment, and selective reporting were considered a risk of bias in a few studies only. For four of the nine studies, the risk of bias based on similarity of baseline outcome measurements was unclear. Additional file 9: Table S6 shows the summary of findings table, including the assessment of the quality of the evidence using the GRADE system.

\section{Effect on professional behaviour Referral behaviour}

Figures 3, 4, 5, and 6 show that multifaceted implementation is not more effective than usual care or minimal implementation in improving guideline concordant referral behaviour. The statistical heterogeneity of the pooled studies is high.

\section{Treatment behaviour}

Figures 7, 8, and 9 show that there is no statistically significant difference between multifaceted implementation and usual care or minimal implementation in providing adequate patient information and prescribing medication. However, active treatment was more often advised in the multifaceted implementation groups than in the control groups (Fig. 9, OR 0.69; $95 \%$ CI 0.48 to 0.99).

\section{Effect on patient outcomes}

Additional file 10: Table S7 shows the results for patient outcomes at 12-month follow-up. Three studies reported outcomes on patient level $[20,23,30]$. The most common patient outcomes measured were functional capacity or disability, days of sick leave, and quality of life (QoL). No significant differences on 12-month follow-up were found for any of these outcomes.

\section{Effect on cost of care}

Three studies performed and separately reported a cost-effectiveness analysis for their implementation strategies [21, 24, 29]. Two of these cost-effectiveness

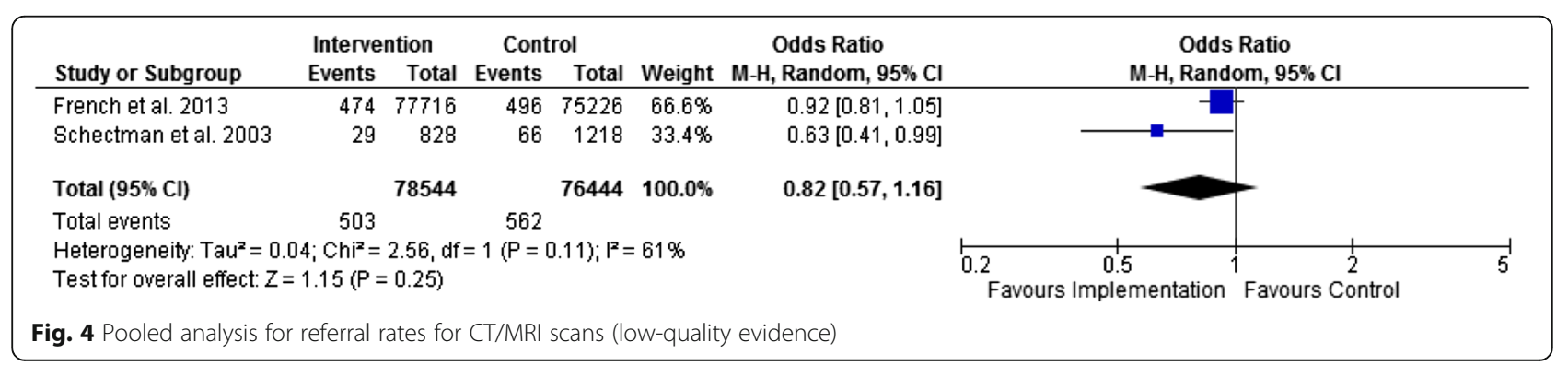




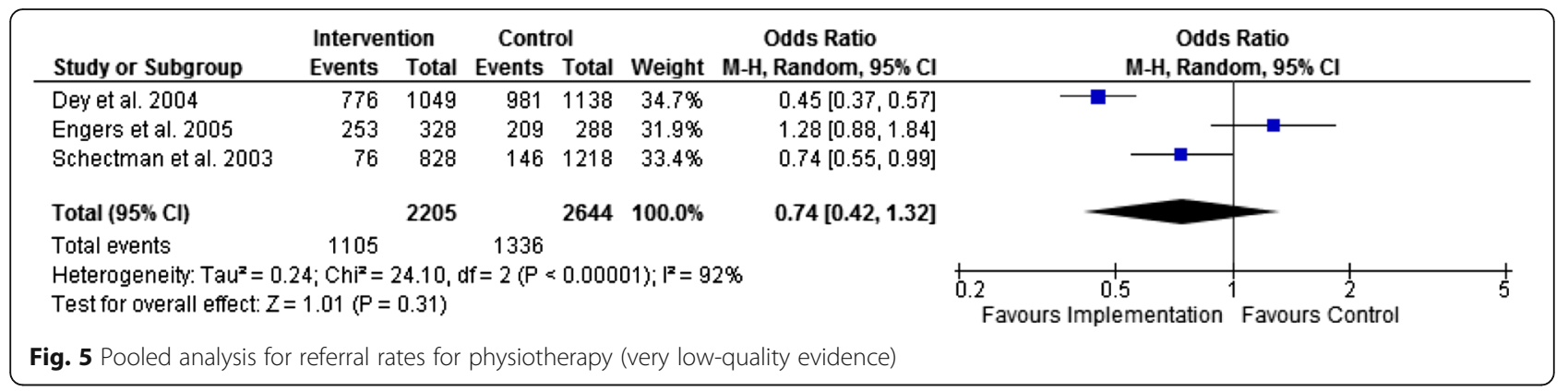

analyses $[21,29]$ showed that multifaceted implementation yielded lower costs and more effects, although these results were not statistically significant. The third study showed no cost or effect advantages for the intervention group compared to the control group.

\section{Discussion}

This review showed that multifaceted strategies for the implementation of neck and/or back pain guidelines in health care do not significantly improve professional behaviour outcomes. Only active treatment was more often advised in the multifaceted implementation groups than in the control groups. No effects on patient outcomes or cost of care could be found. These results are not in line with findings from previous research in other fields that showed that active, multifaceted implementation strategies are effective in changing professional behaviour [63-69] compared to passive dissemination of guidelines or minimal implementation activities. However, the results are in line with a more recent and more elaborate overview of systematic reviews, which suggested that multifaceted implementation strategies are not more effective than other strategies [11].

Few studies that were found in the electronic database search were included in the current review. Many studies were excluded because their interpretations of 'multifaceted' strategies were not in line with the EPOC definition. For example, several studies indicated having applied a multifaceted approach by organizing several workshops on multiple occasions. However, all workshops were part of one element, i.e. educational meetings. As according to the EPOC taxonomy, an intervention is multifaceted if it applies two or more elements [12], these strategies did not meet the criteria for being multifaceted. It seems that either the EPOC taxonomy is not often used or the definition of multifaceted strategies is open to interpretation. Either way, consensus on the definition of multifaceted and application of the taxonomy could improve insight into the effectiveness of multifaceted implementation strategies.

Only 12 articles were identified and included in this study. These studies were not able to produce highquality evidence for changes on professional or patient outcomes in the current review. Only 3 articles reported cost-effectiveness analyses of original studies. These cost-effectiveness studies were evaluated by a recent review by Jensen et al., who showed that the quality of these economic evaluations was moderate and that the studies, although similar to one another, showed conflicting results on cost-effectiveness [70]. It is advisable that researchers implementing guidelines also include cost-effectiveness in their analyses. Multifaceted implementation strategies can be costly, especially when they are applied to implement guidelines on a national level. The efforts and resources for applying these strategies are only worthwhile if they are effective in improving patient outcomes or quality of care. If these strategies also lead to changes in specific professional behaviour, e.g. less unnecessary healthcare utilization referrals or medication prescriptions, and advises to return-to-work, the costs of implementation might be offset by the decrease in costs of care.

Of the included studies, only 3 applied a patientmediated implementation element. Of these 3 studies,

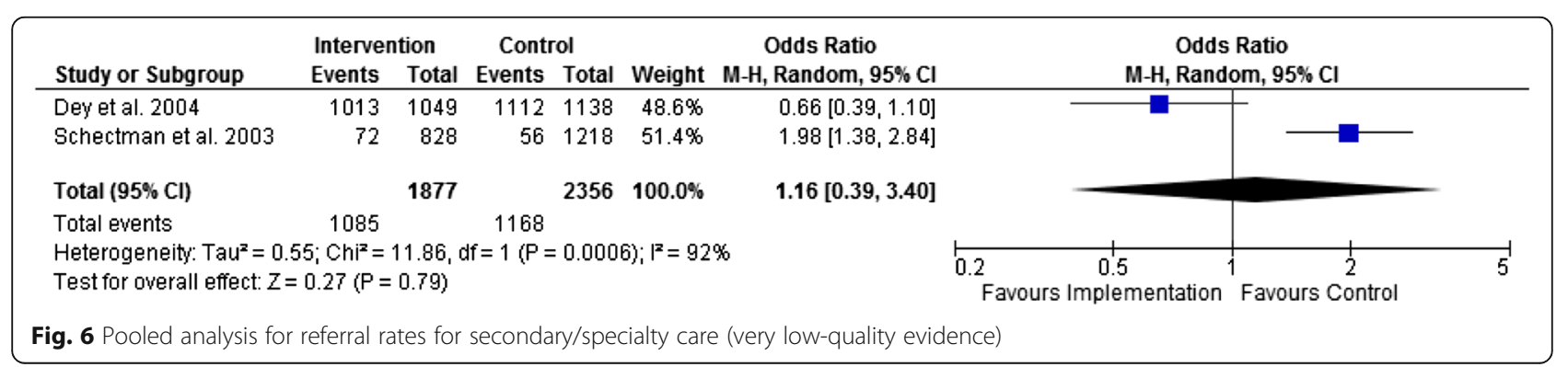




\begin{tabular}{|c|c|c|c|c|c|c|c|c|c|}
\hline Study or Subgroup & \multicolumn{2}{|c|}{ Implementation } & \multicolumn{2}{|c|}{ Control } & Weight & $\begin{array}{c}\text { Odds Ratio } \\
\text { M-H, Random, } 95 \% \mathrm{Cl}\end{array}$ & \multicolumn{3}{|c|}{$\begin{array}{c}\text { Odds Ratio } \\
\mathrm{M}-\mathrm{H}, \text { Random, } 95 \% \mathrm{Cl}\end{array}$} \\
\hline Bekkering et al. 2005(a) & 18 & 247 & 32 & 253 & $26.1 \%$ & $0.54[0.30,1.00]$ & $\rightarrow$ & & \\
\hline Bishop et al. 2006 (1) & 146 & 162 & 139 & 149 & $17.4 \%$ & $0.66[0.29,1.50]$ & & & \\
\hline Bishop et al. 2006 (2) & 142 & 151 & 139 & 149 & $14.5 \%$ & $1.14[0.45,2.88]$ & & & \\
\hline Engers et al. 2005 & 86 & 328 & 69 & 288 & $41.9 \%$ & $1.13[0.78,1.63]$ & & & \\
\hline Total $(95 \% \mathrm{Cl})$ & & 888 & & 839 & $100.0 \%$ & $0.85[0.57,1.27]$ & & & \\
\hline Total events & 392 & & 379 & & & & & & \\
\hline $\begin{array}{l}\text { Heterogeneity: Tau }{ }^{2}=0.0 \\
\text { Test for overall effect: } Z=\end{array}$ & $\begin{array}{l}\mathrm{Chi}^{2}=4.9 \\
80(\mathrm{P}=0 .\end{array}$ & $d f=3($ & $(P=0.18)$ & $i^{2}=30$ & & & $\begin{array}{|cc|}0.2 & 0.5 \\
\text { Favours Implementation }\end{array}$ & $\begin{array}{c}2 \\
2 \\
\text { Favours Control }\end{array}$ & 5 \\
\hline
\end{tabular}

only 1 actually measured patient outcomes (20). Two other studies reported patient outcomes, while they had not applied patient-mediated elements. This might be an explanation for the lack of effect on patient outcomes. Grol and Wensing [7] identified several patient characteristics as possible factors for implementation success. For example, patient attitude and knowledge might pose a barrier for the uptake of changes by professionals. Therefore, when aiming to improve professional practice, applying elements that are targeted at patientmediated barriers and facilitators might be essential to guideline implementation. This is underlined by the slightly more positive results in the study of Becker et al. [20] compared to the other studies that reported patient outcomes. However, merely applying patient-mediated interventions does not necessarily address patient barriers. It is advisable that these barriers be taken into account when designing implementation strategies.

Regarding the effectiveness of more comprehensive strategies compared to strategies that apply fewer elements, the results from the current review are inconclusive. It seems that more does not always mean better, and multifaceted strategies possibly are only more effective when they apply different elements that are targeted at various barriers and facilitators for change [71]. However, this was not confirmed in our review. A recent review by Mesner et al. suggested that the success of implementation interventions does not necessarily depend on the specific type of interventions but rather might be determined by the increase of frequency and duration of implementation interventions [72]. There is still a lack of the use of theory in implementation research, and studies on guideline implementation strategies poorly justify the choice of intervention [72, 73]. This is in line with the findings of the current review, in which only four studies (partly) based their strategies on theories and also reported on the development of their strategy. This could be one possible explanation for the lack of effective results in these studies [73]. However, for many studies, it is unknown whether the implementation strategies were conducted as planned, which might be another factor influencing the effectiveness of the implementation strategies. Process evaluations are necessary to gain more insight into this factor; however, of the included studies in this review, only one study performed a process evaluation [28] and reported moderate to high levels of fidelity [74].

\section{Strengths and limitations of this study}

When interpreting the results of the current review, some limitations should be taken into account. Firstly, this review did not search for unpublished studies. Also, due to the amount of synonyms for the term 'Implementation' included in the search strategy, it might be possible that some studies were missed during the search phase. Furthermore, all but two studies were published before 2007, and the quality of the evidence found in these studies was very low to moderate, according to the GRADE assessment performed in the

\begin{tabular}{|c|c|c|c|c|c|c|c|c|c|}
\hline Study or Subgroup & \multicolumn{2}{|c|}{ Implementation } & \multicolumn{2}{|c|}{ Control } & Weight & $\begin{array}{c}\text { Odds Ratio } \\
\text { M-H, Random, } 95 \% \mathrm{Cl}\end{array}$ & \multicolumn{3}{|c|}{$\begin{array}{c}\text { Odds Ratio } \\
\text { M-H, Random, } 95 \% \mathrm{Cl}\end{array}$} \\
\hline Bishop et al. 2006 (1) & 24 & 162 & 34 & 149 & $10.0 \%$ & $0.59[0.33,1.05]$ & 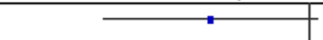 & 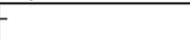 & \\
\hline Bishop et al. 2006 (2) & 29 & 151 & 34 & 149 & $10.8 \%$ & $0.80[0.46,1.40]$ & & & \\
\hline Dey et al. 2004 & 196 & 1049 & 213 & 1138 & $51.8 \%$ & $1.00[0.80,1.24]$ & - & - & \\
\hline Engers et al. 2005 & 198 & 328 & 188 & 288 & $27.4 \%$ & $0.81[0.58,1.13]$ & 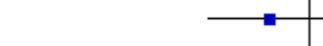 & & \\
\hline Total (95\% Cl) & & 1690 & & 1724 & $100.0 \%$ & $0.87[0.72,1.06]$ & & & \\
\hline Total events & 447 & & 469 & & & & & & \\
\hline $\begin{array}{l}\text { Heterogeneity: Tau }{ }^{2}=0 \\
\text { Test for overall effect: } z\end{array}$ & $\begin{array}{l}01 ; \mathrm{Chi}^{2}= \\
=1.40(\mathrm{P}=\end{array}$ & $\begin{array}{l}50, d f= \\
16)\end{array}$ & $3(P=0.3$ & ;2); $\left.\right|^{2}=$ & $=14 \%$ & & $\begin{array}{|cc|}0.2 & 0.5 \\
\text { Favours Implementation }\end{array}$ & $\begin{array}{c}2 \\
2 \\
\text { Favours Control }\end{array}$ & 5 \\
\hline
\end{tabular}




\begin{tabular}{|c|c|c|c|c|c|c|c|c|c|}
\hline \multirow[b]{2}{*}{ Study or Subgroup } & \multicolumn{2}{|c|}{ Implementation } & \multicolumn{2}{|c|}{ Control } & \multirow[b]{2}{*}{ Weight } & \multirow{2}{*}{$\begin{array}{c}\text { Odds Ratio } \\
\text { M-H, Random, } 95 \% \mathrm{Cl}\end{array}$} & \multirow{2}{*}{\multicolumn{3}{|c|}{$\begin{array}{c}\text { Odds Ratio } \\
\text { M-H, Random, } 95 \% \mathrm{Cl}\end{array}$}} \\
\hline & Events & Total & Events & Total & & & & & \\
\hline Bekkering et al. $2005(\mathrm{a})$ & 64 & 247 & 99 & 253 & $26.9 \%$ & $0.54[0.37,0.80]$ & $\longrightarrow$ & & \\
\hline Bishop et al. 2006 (1) & 100 & 162 & 85 & 149 & $24.0 \%$ & $1.21[0.77,1.91]$ & & & \\
\hline Bishop et al. 2006 (2) & 71 & 151 & 85 & 149 & $23.9 \%$ & $0.67[0.42,1.05]$ & & & \\
\hline Engers et al. 2005 & 44 & 328 & 65 & 288 & $25.2 \%$ & $0.53[0.35,0.81]$ & & & \\
\hline Total $(95 \% \mathrm{Cl})$ & & 888 & & 839 & $100.0 \%$ & $0.69[0.48,0.99]$ & & & \\
\hline Total events & 279 & & 334 & & & & & & \\
\hline $\begin{array}{l}\text { Heterogeneity: } \operatorname{Tau}^{2}=0.0 \\
\text { Test for overall effect: } Z=\end{array}$ & $\begin{array}{l}\mathrm{Chi}^{2}=8.9 \\
99(\mathrm{P}=0.0\end{array}$ & $d f=3(F$ & $P=0.03)$ & $; z^{2}=66$ & & & $\begin{array}{cc}0.2 & 0.5 \\
\text { Favours Implementatior }\end{array}$ & 1 Favours Control & 5 \\
\hline
\end{tabular}

current review. Another important limitation is the comparability of the studies. Not only did the studies apply various implementation strategies, usual dissemination in the control groups also varied. Besides, there was a wide variation in the outcomes that were measured, how they were measured, and how they were reported. In the meta-analyses, the statistical heterogeneity was large, and results should therefore be interpreted with caution. For example, one study where the control group did not receive any implementation strategy was compared with a study in which the control group received a patient-mediated implementation strategy. Other reasons for heterogeneity might be clinical (e.g. due to different settings and patients) or statistical (e.g. different study sizes). No sensitivity analyses were performed due to the small number of studies that could be included in the meta-analyses, and notwithstanding the high statistical heterogeneity found in these analyses as expressed by the $I^{2}$ measures, these analyses might give an insight into the effect directions of the included studies.

By following the method guidelines for systematic reviews as posed by the Cochrane Back Review Group [75], and the Cochrane Handbook for Systematic Reviews of Interventions [12], the current review pursued the highest methodological quality. By applying a broad and comprehensive search strategy and supplementary hand search of reference lists of included studies, this review ensured that as few as possible, eligible studies were missed. To further minimize this chance, no language restrictions were applied during the inclusion and data collection phases.

\section{Conclusion}

This review showed that multifaceted strategies for the implementation of neck and/or back pain guidelines in health care do not significantly improve professional behaviour outcomes. Only active treatment was more often advised in the multifaceted implementation groups than in the control groups. No effects on patient outcomes or cost of care could be found.

\section{Additional files}

\section{Additional file 1: Appendix A. Full electronic database searches.} (DOCX $14 \mathrm{~kb}$ )

Additional file 2: Appendix C. Data sources and calculations for meta-analyses. (DOCX $15 \mathrm{~kb}$ )

Additional file 3: Table S1. References to included studies. (DOCX $15 \mathrm{~kb}$ )

Additional file 4: Table S2. References to excluded full-text studies. (DOCX $17 \mathrm{~kb}$ )

Additional file 5: Table S3. Characteristics of included studies. (DOCX 23 kb)

Additional file 6: Appendix B. List of excluded studies and reasons for exclusion. (DOCX $17 \mathrm{~kb}$ )

Additional file 7: Table S4. Interventions of included studies according to EPOC taxonomy (all on professional level unless stated otherwise). (DOCX $15 \mathrm{~kb}$ )

Additional file 8: Table S5. Grouped outcome measures of included studies (all on professional level unless stated otherwise). (DOCX $14 \mathrm{~kb}$ )

Additional file 9: Table S6. Summary of findings. (DOCX 17 kb)

Additional file 10: Table S7. Patient outcomes at 12-month follow-up. (DOCX $14 \mathrm{~kb}$ )

\section{Abbreviations}

CEA: Cost-effectiveness analysis; CT: Computed tomography; EPOC: Effective Practice and Organisation of Care; GP: General practitioner; LBP: Low back pain; MRI: Magnetic resonance imaging; NP: Neck pain; NPs: Nurse practitioners; PAs: Physician assistants; PT: Physiotherapist; QoL: Quality of life; $\mathrm{RCT}$ : Randomized controlled trial; $X$-ray: Röntgen radiation imaging

\section{Acknowledgements}

The authors thank Linda Schoonmade, a medical information specialist at the VU University medical centre Amsterdam, for her assistance in the development of the search strategy.

\section{Funding}

This systematic review was part of a project funded by The Netherlands Organisation for Health Research and Development (ZonMw).

\section{Authors' contributions}

All authors collaborated in conceiving this study. AS and MD screened citations and full-text articles and extracted, analysed, and interpreted the data. AS wrote the initial manuscript for this study. All authors were involved in the interpretation of the data and critical revision of the manuscript. All authors read and approved the final manuscript.

\section{Competing interests}

The authors declare that they have no competing interests.

Consent for publication

Not applicable. Data and materials are available for reviewers upon request. 


\section{Ethics approval and consent to participate}

Not applicable.

\section{Author details}

'Department of Public and Occupational Health, VU University medical centre and the EMGO+ Institute for Health and Care Research, PO Box 7057, 1007, MB, Amsterdam, The Netherlands. ${ }^{2}$ Department of Health Sciences, Faculty of Earth and Life Sciences, VU University, Amsterdam, The Netherlands. ${ }^{3}$ Research Centre for Insurance Medicine, Collaboration between AMC-UMCG-UWV-VUmc, Amsterdam, The Netherlands.

Received: 31 March 2016 Accepted: 15 August 2016 Published online: 20 September 2016

\section{References}

1. Vos T, Flaxman AD, Naghavi M, Lozano R, Michaud C, Ezzati M, et al. Years lived with disability (YLDs) for 1160 sequelae of 289 diseases and injuries 1990-2010: a systematic analysis for the Global Burden of Disease Study 2010. Lancet. 2012;380(9859):2163-96.

2. Hoy D, March L, Brooks P, Blyth F, Woolf A, Bain C, et al. The global burden of low back pain: estimates from the Global Burden of Disease 2010 study. Ann Rheum Dis. 2014;73:968-74.

3. Hoy D, March L, Woolf A, Blyth F, Brooks P, Smith E, et al. The global burden of neck pain: estimates from the global burden of disease 2010 study. Ann Rheum Dis. 2014;73(7):1309-15.

4. Koes BW, van Tulder M, Lin CW, Macedo LG, McAuley J, Maher C. An updated overview of clinical guidelines for the management of non-specific low back pain in primary care. Eur Spine J. 2010;19(12):2075-94.

5. Childs JD, Cleland JA, Elliott JM, Teyhen DS, Wainner RS, Whitman JM, et al. Neck pain: clinical practice guidelines linked to the international classification of functioning, disability, and health from the orthopaedic section of the American Physical Therapy Association. J Orthop Sports Phys Ther. 2008;38(9):A1-A34.

6. The Institute of Medicine. Clinical practice guidelines we can trust Washington: The Institute of Medicine; 2011.

7. Grol R, Wensing M. Improving patient care: the implementation of change in health care (in Dutch: Implementatie: effective verbetering van de patiëntenzorg). Amsterdam: Reed Business Education; 2013.

8. Forsetlund L, Bjørndal A, Rashidian A, Jamtvedt G, O'Brien MA, Wolf FM, et al. Continuing education meetings and workshops: effects on professional practice and health care outcomes. Cochrane Database Syst Rev. 2009. doi: 10.1002/14651858.CD003030.pub2.

9. O'Brien MA, Rogers S, Jamtvedt G, Oxman AD, Odgaard-Jensen J, Kristoffersen DT, et al. Educational outreach visits: effects on professional practice and health care outcomes. Cochrane Database Syst Rev. 2007. doi:10.1002/14651858.CD000409.pub2.

10. Jamtvedt G, Young JM, Kristoffersen DT, O'Brien MA, Oxman AD. Audit and feedback: effects on professional practice and health care outcomes. Cochrane Database Syst Rev. 2006. doi:10.1002/14651858.CD000259.pub2.

11. Squires JE, Sullivan K, Eccles MP, Worswick J, Grimshaw JM. Are multifaceted interventions more effective than single-component interventions in changing health-care professionals' behaviours? An overview of systematic reviews. Implement Sci. 2014;9(1):152

12. Effective Practice and Organisation of Care (EPOC). EPOC taxonomy. 2015. https://epoc.cochrane.org/epoc-taxonomy. Accessed 14 June 2016.

13. Higgins JPT, Green S (editors). Cochrane Handbook for Systematic Reviews of Interventions version 5.1.0 [updated March 2011]. The Cochrane Collaboration, 2011. http://handbook.cochrane.org/ Last accessed 27 Jan 2016.

14. Cochrane Effective Practice and Organisation of Care Review Group. Suggested risk of bias criteria for EPOC reviews. https://epoc.cochrane.org/ sites/epoc.cochrane.org/files/uploads/Suggested\%20risk\%20of\%20bias\% 20criteria\%20for\%20EPOC\%20reviews.pdf. Accessed 23 Feb 2016.

15. Kamper SJ, Apeldoorn AT, Chiarotto A, Smeets RJEM, Ostelo RWJG, Guzman J, et al. Multidisciplinary biopsychosocial rehabilitation for chronic low back pain: Cochrane systematic review and meta-analysis. BMJ. 2015;350:h444

16. Boluyt N, Rottier BL, Langendam MW. Guidelines are made more transparent with the GRADE method: considerations for recommendations are explicit in the new method. Ned Tijdschr Geneeskd. 2012;156:1-7.

17. Guyatt $G H$, Oxman AD, Vist GE, Kunz R, Falck-Ytter Y, Schünemann HJ. GRADE: what is 'quality of evidence' and why is it important to clinicians? BMJ. 2008:336:995-8.
18. Guyatt GH, Oxman AD, Vist GE, Kunz R, Falck-Ytter Y, Alonso-Coello P, et al. GRADE: an emerging consensus on rating quality of evidence and strength of recommendations. BMJ. 2008;336:924-6.

19. Schünemann HJ, Jaeschke R, Cook DJ, Bria WF, El-Sohl AA, Ernst A, et al. An official ATS statement: grading the quality of evidence and strength of recommendations in ATS guidelines and recommendations. Am J Respir Crit Care Med. 2006;174:605-14.

20. Becker A, Leonhardt C, Kochen MM, Keller S, Wegscheider K, Baum E, et al. Effects of two guideline implementation strategies on patient outcomes in primary care. A cluster randomized controlled trial. SPINE. 2008;33(5):4732-480.

21. Becker A, Held H, Redaelli M, Chenot JF, Leonhardt C, Keller S, et al. Implementation of a guideline for low back pain management in primary care. A cost-effectiveness analysis. SPINE. 2012;37(8):701-10.

22. Bekkering GE, Hendriks HJM, van Tulder MW, Knol DL, Hoeijenbos M, Oostendorp RAB. Effect on the process of care of an active strategy to implement clinical guidelines on physiotherapy for low back pain: a cluster randomised controlled trial. Qual Saf Health Care. 2005;14:107-12.

23. Bekkering GE, van Tulder MW, Hendriks HJM, Koopmanschap MA, Knol DL, Bouter LM, et al. Implementation of clinical guidelines on physical therapy for patients with low back pain: randomized trial comparing patient outcomes after a standard and active implementation strategy. Phys Ther. 2005;85:544-55.

24. Hoeijenbos M, Bekkering T, Lamers L, Hendriks E, van Tulder M, Koopmanschap M. Cost-effectiveness of an active implementation strategy for the Dutch physiotherapy guideline for low back pain. Health Policy. 2005;75:85-98.

25. Bishop PB, Wing PC. Knowledge transfer in family physicians managing patients with acute low back pain: a prospective randomized control trial. Spine J. 2006;6:282-8.

26. Dey P, Simpson CWR, Collins SI, Hodgson G, Dowrick CF, Simison AJM, et al. Implementation of RCGP guidelines for acute low back pain: a cluster randomised controlled trial. Br J Gen Pract. 2004;54:33-7.

27. Engers AJ, Wensing $M$, van Tulder MW, Timmermans A, Oostendorp RAB, Koes BW, et al. Implementation of the Dutch low back pain guideline for general practitioners. SPINE. 2005;6:595-600.

28. French SD, McKenzie JE, O'Connor DA, Grimshaw JM, Mortimer D, Francis J, et al. Evaluation of a theory-informed implementation intervention for the management of acute low back pain in general medical practice: the IMPLEMENT cluster randomised trial. PLoS ONE. 2013;8(6):e65471. doi:10.1371/journal.pone.0065471.

29. Mortimer D, French SD, McKenzie JE, O'Connor DA, Green SE. Economic evaluation of active implementation versus guideline dissemination for evidence-based care of acute low-back pain in a general practice setting. PLoS ONE. 2013;8(10):e75647. doi:10.1371/journal.pone.0075647.

30. Rebbeck T, Maher CG, Refshauge KM. Evaluation two implementation strategies for whiplash guidelines in physiotherapy: a cluster-randomised trial. Aust J Physiother. 2006;52:165-74.

31. Schectman JM, Schroth WS, Verme D, Voss JD. Randomized controlled trial of education and feedback for implementation of guidelines for acute low back pain. J Gen Intern Med. 2003;18:773-80.

32. Ammendolia C, Hogg-Johnson S, Pennick V, Glazier R, Bombardier C. Implementing evidence-based guidelines for radiography in acute low back pain: a pilot study in a chiropractic community. J Manip Physiol Ther. 2003;27(3):170-9.

33. Bussières $A$, Laurencelle $L$, Peterson $C$. Diagnostic imaging guidelines implementation study for spinal disorders. A randomized trial with postal follow-ups. J Chiropr Educ. 2010; 24(1):2-18.

34. Bekkering GE, Hendriks HJM, Van Tulder MW. Effectiviteit van een actieve implementatiestrategie van de KNGF-richtlijn 'Lage-rugpijn'. Ned Tijdschr Fysiother. 2005;115(3):62-7.

35. Cherkin D, Deyo RA, Berg AO, Bergman JJ, Lishner DM. Evaluation of a physician education intervention to improve primary care for low-back pain 1: impact on physicians. SPINE. 1991;16(10):1168-72.

36. Cunningham CG, Flynn TA, Toole CM, Ryan RG, Gueret PWJ, Bulfin S, et al. Working backs project-implementing low back pain guidelines. Occup Med. 2008:58:580-3.

37. Taramona Espinoza CP, Skupin M, Montezuma D, Sandouk Z, Drake S. Adherence to guidelines for low back pain imaging: comparison of a teaching and non-teaching clinic. Abstracts from the $35^{\text {th }}$ Annual Meeting of the Society of General Internal Medicine. JGIM. 2012;27 Suppl 2:S99-574. doi:10.1007/s11606-012-2038-0. 
38. Evans DW, Foster NE, Underwood M, Vogel S, Breen AC, Pincus T. Testing the effectiveness of an innovative information package on practitioner reported behaviour and beliefs: the UK Chiropractors, Osteopaths and Musculoskeletal Physiotherapists Low back pain ManagemENT (COMPLeMENT) trial. BMC Musculoskelet Disord. 2005;6(41); doi:10.1186/1471-2474-6-41.

39. Fleuren M, Dusseldorp E, Van den Bergh S, Vlek H, Wildschut J, Van den

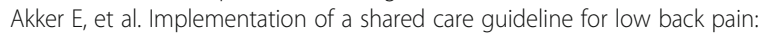
effect on unnecessary referrals. Int J Qual Health Care. 2010;22(5):415-20.

40. Jensen CE, Riis A, Pedersen KM, Jensen MB, Petersen KD. Study protocol of an economic evaluation of an extended implementation strategy for the treatment of low back pain in general practice: a cluster randomised controlled trial. Implement Sci. 2014;9:140.

41. Lang E, Kastner S, Liebig K, Neundörfer B. Interventions for improvement of primary care in patients with low back pain: how effective are advice to primary care physicians on therapies and a multimodal therapy program arising out of cooperation of outpatient health care structures? Schmerz. 2002;16:22-33.

42. McKenzie JE, French SD, O'Connor DA, Grimshaw JM, Mortimer D, Michie S, et al. IMPLEmenting a clinical practice guideline for acute low back pain evidence-based management in general practice (IMPLEMENT): cluste randomised controlled trial study protocol. Implement Sci. 2008;3:11. doi:10.1186/1748-5908-3-11.

43. McKenzie JE, O'Connor DA, Page MJ, Mortimer SD, French SD, Walker BF. Improving the care for people with acute low-back pain by allied health professionals (the ALIGN trial): a cluster randomised trial protocol. Implement Sci. 2013;5(86). doi:10.1186/1748-5908-5-86.

44. Mortimer D, French SD, McKenzie JE, O'Connor DA, Green SE. Protocol for economic evaluation alongside the IMPLEMENT cluster randomised controlled trial. Implement Sci. 2008:3:12. doi:10.1186/1748-5908-3-12.

45. Rasmussen F $\varnothing$. Kunnskapsbasert ryggomsorg - en pilotstudie om etterutdanning av allmennleger. Tidsskr Nor Laegeforen. 2002;122:1794-6.

46. Rebbeck TJ, Refshauge KM, Maher CG. Use of clinical guidelines for whiplash by insurers. Aust Health Rev. 2006;30(4):442-9.

47. Rebbeck T, Stewart M, Cameron I, Stewart J. Treatment of chronic whiplash: a systematic review and clinical guidelines. Physiotherapy. 2011;97:s1.

48. Rebbeck T, Macedo LG, Maher CG. Compliance with clinical guidelines for whiplash improved with a targeted implementation strategy: a prospective cohort study. BMC Health Serv Res. 2013;13(213). doi:10.1186/1472-6963-13-213.

49. Rebbeck T, Macedo L, Paul P, Trevena L, Camron ID. General practitioners' knowledge of whiplash guidelines improved with online education. Aust Health Rev. 2013;37:688-94.

50. Richings K, Taylor C, Morris J. Changing GPS guideline adherence in relation to ordering plain lumbar spine X-rays for NSLBP: the role of the physiotherapist. Physiotherapy. 2011;97:s1.

51. Riis A, Jensen CE, Bro F, Maindal HT, Petersen KD, Jensen MB. Enhanced implementation of low back pain guidelines in general practice: study protocol of a cluster randomised controlled trial. Implement Sci. 2013;8(124). doi:10.1186/1748-5908-8-124

52. Rossignol M, Abenhaim L, Séguin P, Neveu A, Collet JP, Ducruet T, et al. Coordination of primary health care for back pain: a randomized controlled trial. SPINE. 2000;25(2):251-9.

53. Rutten G, Harting J, Bartholomew LK, Oostendorp RAB, De Vries NK. Results of the pilot study of a multilevel intervention to improve adherence to evidence based guidelines for low back pain. Physiotherapy. 2011;97:s1.

54. Rutten GM, Harting J, Bartholomew LK, Braspenning J, Van Dolder R, Heijmans MFGJ, et al. Development of a theory- and evidence-based intervention to enhance implementation of physical therapy guidelines for the management of low back pain. Arch Public Health. 2014;72(1). doi:10. 1186/2049-3258-72-1.

55. Sandner-Kiesling A, Gspurning E, Granitz GG, Thalhammer G. "Rückenschmerz.ade" — a disease management project for the implementation of guidelines in the treatment of chronic low back pain. Eur J Pain. 2009;13:S55-S285.

56. Shenoy S. Cluster randomized controlled trial to evaluate the effectiveness of a multifaceted active strategy to implement low back pain practice guidelines: effect on competence, process of care and patient outcomes in physical therapy. Dissertation at University of Pittsburgh, School of Health and Rehabilitation Sciences, 2013.

57. Slater H, Briggs AM, Smith AJ, Bunzli S, Davies SJ, Quintner JL. Implementing evidence-informed policy into practice for health care professionals managing people with low back pain in Australian rural settings: a preliminary prospective single-cohort study. Pain Med. 2014;15:1657-68.
58. Stiell IG, Clement CM, Grimshaw J, Brison RJ, Rowe BH, Schull MJ, et al. Implementation of the Canadian C-spine rule: a prospective 12 centre cluster randomised trial. BMJ. 2009;339:b4146.

59. Suman A, Schaafsma FG, Elders PJM, Van Tulder MW, Anema JR. Costeffectiveness of a multifaceted implementation strategy for the Dutch multidisciplinary guideline for nonspecific low back pain: design of a stepped-wedge cluster randomised controlled trial. BMC Public Health. 2015;15:522. doi:10.1186/s12889-015-1876-1.

60. Tracey NG, Martin JB, McKinstry CS, Mathew BM. Guidelines for lumbar spine radiography in acute low back pain: effect of implementation in an accident and emergency department. Ulster Med J. 1994;63(1):12-7.

61. Twomey P. Making the best use of a radiology department: an example of implementation of a referral guideline within a primary care organisation. Qual Prim Care. 2003;11:53-9.

62. Van Dulmen SA, Maas MJ, Staal B, Rutten G, Kiers H, Nijhuis-Van der Sanden $M$, et al. Effectiveness of peer assessment for implementing a Dutch physical therapy low back pain guideline: cluster randomized controlled trial. Phys Ther. 2014;94:1396-409.

63. Hakkennes S, Dodd K. Guideline implementation in allied health professions: a systematic review of the literature. Qual Saf Health Care. 2008;17:296-300.

64. Grol R, Grimshaw J. From best evidence to best practice: effective implementation of change in patients' care. Lancet. 2003;362:1225-30.

65. Grol R, Wensing M, Eccles $M$. Improving patient care. The implementation of change in clinical practice. Edinburgh: Elsevier Butterworth Heineman; 2005.

66. Prior M, Guerin M, Grimmer-Somers K. The effectiveness of clinical guideline implementation strategies-a synthesis of systematic review findings. J Eval Clin Pract. 2008;14:888-97.

67. Francke AL, Smit MC, de Veer AJ, Mistiaen P. Factors influencing the implementation of clinical guidelines for health care professionals: a systematic meta-review. BMC Med Inform Dec Mak. 2008;8:38.

68. Boaz A, Baeza J, Fraser A, European Implementation Score Collaborative Group (EIS). Effective implementation of research into practice: an overview of systematic reviews of the health literature. BMC Res Notes. 2011;4:212. doi:10.1186/1756-0500-4-212

69. Grimshaw JM, Eccles MP, Lavis JN, Hill SJ, Squires JE. Knowledge translation of research findings. Implement Sci. 2012;7:50.

70. Jensen $C E$, Jensen MB, Riis A, Petersen $K D$. Systematic review on the cost-effectiveness of implementing guidelines on low back pain management in primary care: is transferability to other countries possible? BMJ Open. 2016;6:e011042. doi:10.1136/bmjopen-2016-011042.

71. Wensing M, Bosch M, Grol R. Developing and selecting interventions for translating knowledge to action. CMAJ. 2010;182(2):E85-88.

72. Mesner SA, Foster NE, French SD. Implementation interventions to improve the management of non-specific low back pain: a systematic review. BMC Musculoskelet Disord. 2016;17:258. doi:10.1186/s12891-016-1110-z.

73. Davies P, Walker AE, Grimshaw JM. A systematic review of the use of theory in the design of guideline dissemination and implementation strategies and interpretation of the results of rigorous evaluations. Implement Sci. 2010;5:14

74. French SD, Green SE, Francis JJ, Buchbinder R, O'Connor DA, Grimshaw JM, et al. Evaluation of the fidelity of an interactive face-to-face educational intervention to improve general practitioner management of back pain. BMJ Open. 2015;5:e007886. doi:10.1136/bmjopen-2015-007886.

75. Furlan AD, Penninck V, Bombardier V, van Tulder M, from the Editorial Board of the Cochrane Back Review Group. SPINE. 2009;34(18):1929-41. 